\title{
A Brief Overview of Robotic Telescopes, Student Research and Education Research in the English Literature
}

Fitzgerald, M. ${ }^{1 *}$, Cutts, R. ${ }^{1,2}$, Salimpour, $S .^{3}$, Slater, S. ${ }^{4}$

\begin{abstract}
In this paper, a brief overview of the 245 known academic publications that can be considered Robotic Telescopes, Student Research and Education related articles is provided. Their method of selection is described as well as their availability on the International Studies of Astronomy Education Research Database (istardb.org) database. The typical types of articles are described and their major locations of publications are outlined. From this a rough impact analysis using a blunt citation metric is used to provide a broad overview of the literature for the field as a whole. These explorations are discussed with broad conclusions presented about how to write an article in the field with some impact as well as where and what to publish as the Robotic Telescopes, Student Research and Education (RTSRE) field continues to grow.

${ }^{1}$ Edith Cowan Institute for Education Research, Edith Cowan University, Joondalup, WA, Australia

2St. Paul's Grammar School, 52 Taylor Rd, Cranebrook NSW 2749 Australia

${ }^{3}$ The University of Melbourne, Victoria, Australia

${ }^{4}$ CAPER Center for Astronomy \& Physics Education Research, USA

*Corresponding author: mfitzasp@gmail.com
\end{abstract}

\section{Introduction}

Astronomy Education Research as a research and practice field has had a steadily growing history (Bailey and Lombardi 2015, Bailey 2011, Lelliott and Rollnick 2010, Bailey and Slater 2003). Much of its development has been heavily in the Astro101 area (Slater et al. 2016, Bretones et al. 2016) where large groups of generally willing subjects are available for study, although significant work has been done in the K-12 domain as well as the planetarium domain.

The first author was involved in two broad reviews of slices of the Astronomy Education Research literature in the last five years, providing a good database in which to explore the whole field of RTSRE (Robotic Telescopes, Student Research and Education) related topics. The first review paper, by Fitzgerald et al. (2014), reviewed Astronomy
Student Research Projects that tried to get students to undertake authentic research in the classroom to some degree. The second paper was Gomez and Fitzgerald (2017), which reviewed the history, development, uses and current state of Robotic Telescopes in Education.

The RTSRE conference was conceived to explore the issues surrounding the three broad fields, Robotic Telescopes, Student Research and Education, represented in the conference name. While there have been numerous people working in the field over the past couple of decades, they have all been situated in a variety of different institutions, fields and endeavours. Some of these people are in astronomy departments, some in education departments, some in teaching only institutions, some as individual researchers and practitioners unlinked to a major institution.

The RTSRE field does not fit easily into any one 
locale or institution and has members who cross boundaries between many fields. This makes finding relevant literature problematic as there has been no small bounded set of literature collections where these can easily be found. Astronomy Education Research had its own central journal (Astronomy Education Review) for 13 years (Fraknoi, 2014) until 2013, when it was retired by the American Astronomical Society (AAS). But even then, authors did largely continue to submit their higher impact articles to higher impact journals in their own fields.

As the RTSRE field is made up of many people who are in diverse academic (and diverse non-academic) fields and the field itself is lacking an obvious central journal for the moment, providing a bibliometric overview of the literature seemed to be a helpful and useful task to help the development of the field.

\section{The Continuing Search for} RTSRE-Related Articles and Their Selection

Astronomy, as a field, has a small total number of journals (85 in Scopus) and only a few of which are obvious locations for cutting edge research. Most professional astronomers will likely be able to recite at least $90 \%$ of the top quartile of astronomy journals. Education is different, there are 1066 journals listed in Scopus. In those tomes (largely online nowadays) there is a vast swath of physics and astronomy relevant literature. Add to this numerous conference proceedings locations (sometimes on websites for one-off conferences) and numerous published books in different fields and the endeavour to find relevant articles can quickly become overwhelming to any researcher, novice or expert.

This challenge of finding relevant literature is a bigger problem than it may seem at first. Whether research-driven, education-driven or practitioner-driven, any endeavour should be informed by others' work. What could be more disappointing than to spend a few on years a project, only to discover that somebody else had done it already and found it wasn't very useful. If you were attempting to run a project that inspired students, or helped them learn in some manner using telescopes, then reading and understanding the literature on what makes students tick in general and then also what has been tried, worked or failed in the past would be indispensable preliminary reading. If higher level, methodologically robust, education research is your goal, then is absolutely essential to know the current state of the field. Literature reviews such as these have not been easily achievable.

To help address this problem, the RTSRE-relevant literature abstracts have been uploaded to the istardb.org database. This incorporates a huge chunk of articles found in the literature trawls for two mentioned reviews above as well as another trawl intended specifically for this conference in late 2016 and 2017. The trawl included the last few decades of the major science education journals, astronomy journals, common astronomy education conferences as well as random serendipity.

The set of articles available on istardb.org (which can be accessed by "Browsing by Subject") most likely is the majority of the set of all RTSRE articles in the mainstream literature. It would be quite difficult to put a numerical percentage figure on this though, it was not uncommon that a lead was found to a new set of 5-10 new relevant articles. It is intended that this set of abstracts would asymptotically grow towards $100 \%$ of the RTSRE field as time goes on, and people identify more abstracts. It is a certainly a continual process of collection, of which all researchers are invited to participate in for the enhancement of the field.

A further notable bias is that this has been purely an English language endeavour so far. While it is true that most straight astronomical research, whether theoretical or observational, is written in international journals and conference proceedings and are in English, this is not necessarily the case for scholarly fields outside of astronomy. One of the major astronomy education journals, the "Latin-American Journal of Astronomy Education" (RELEA) (Bretones et al., 2016), is mostly in 


\section{A Brief Overview of Robotic Telescopes, Student Research and Education Research in the \\ English Literature - 3}

Portuguese with some articles in Spanish and a very rare few in English.

There is also a great untapped history of Korean-language astronomy education research in South Korea known to the authors. It is likely that the lack of articles in the RTSRE literature from continental Europe is not due to a lack of activity but a lack of ability of the authors to find articles in the numerous European languages. This is especially true of Masters and Doctoral theses which are typically written in the native language of the country within which the supervising university is located. We do not claim that this article represents the literature as a whole, but certainly is indicative of the English language subset of the literature.

It is acknowledged that there would definitely be a bias in the selected articles. Aside from these conference proceedings themselves (and this being the first issue ever), there is no publication that is purely RTSRE related. All articles are published in journals covering other fields, whether that be Astronomy, Astronomy Education, Science Education, Student Research or Technical Engineering. Most papers are obviously relevant but some could go one way or another depending on who was considering the publication. Therefore, at the margins, the acceptance or rejection of these borderline papers was a line-call that had to be made. The line-call was made by considering whether or not the paper added value to the RTSRE field by its existence.

\section{Typical Articles in the Collection}

While there are many purely astronomy articles where students have been co-authors, these papers have not been included in this literature archive. While they may have arisen out of an RTSRE project or endeavour, they are not truly RTSRE papers, but rather mainstream astronomy research papers.

The distribution of the dates of publication are shown in the histogram in Figure 1. The first thing that strikes one when looking at the distribution is the rapid expansion of publications in the early 1990s. There are a few likely reasons for this. Most of the earliest RTSRE projects, such as the Bradford Robotic Telescope (Baruch, 1992), Telescopes in Education (Clark, 1998), Remote Access Astronomy Project (Lubin and van der Veen, 1992), MicroObservatory (Sadler et al., 2001) and Hands-On Universe (Asbell-Clarke et al., 1996) began at that point in time. It is very likely no coincidence that this times well with the advent of available off-the-shelf, reasonably priced CCDs and computer controlled telescope mounts.

Prior to this early 1990s period, there was less of a focus on education provision and more on getting the technical instrumentation to work reliably (e.g. Genet 2011) let alone provide the telescopes for education use, so there is a lack of education and student research articles during the period. It was, however, during this earlier 1980s period that John Percy (Percy, this proceedings) began his long-sustained astronomy student research career. We admit that some of the earlier more technically focused articles pre-1990 have as yet to be included in the abstract database. These will be entered into the abstract database in due course, but the main publication activity in the field has certainly been since 1990 .

The apparent ebb at the 2017 end of the histogram shown in Figure 1, is largely due to the years being so recent that articles have not been identified as yet, or have not officially gone to press, to go in the abstract database, so the 2017-2018 are likely incomplete as of this publication. The 2015-2016 bin should be complete and representative, although whether the decrease is due to natural fluctuations, due to the ending of the journal "Astronomy Education Review" or due to the 2013 major NASA EPO funding cuts or other such events remains to be seen as data for later years becomes available.

The following is a list of example articles to try and illustrate what is considered to be within the scope of the RTSRE field (with aforementioned bias): 


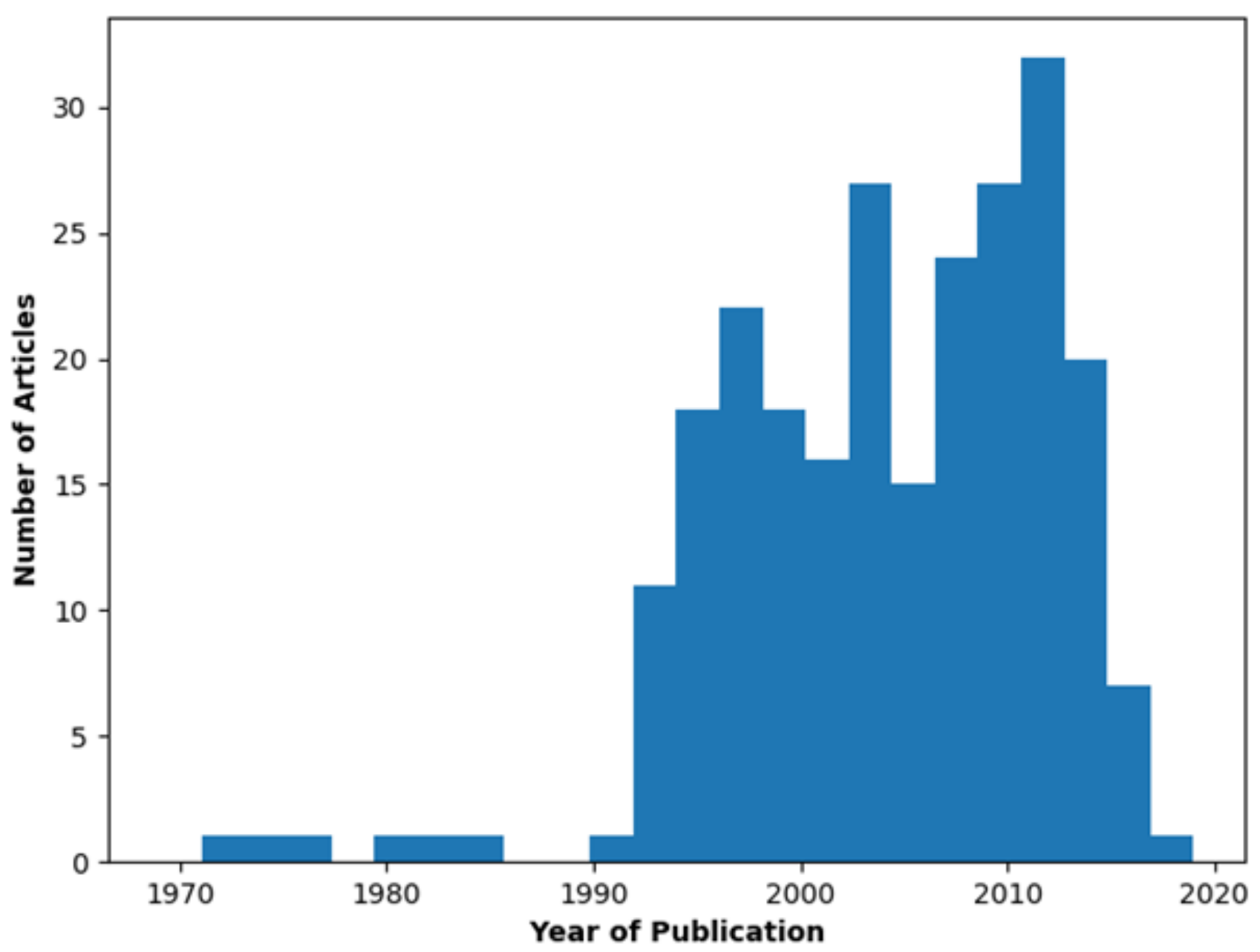

Figure 1. Histogram of papers by year of publication in the iSTARdb collection.

1. Broad Reviews (10 articles)

- Bailey, J.M., (2011) “Astronomy Education Research: Developmental History of the Field and Summary of the Literature" Discussion Paper. National Research Council Board on Science Education.

- Bailey, J.M., Slater, T.F., (2004) “A Review of Astronomy Education Research", Astronomy Education Review, Astronomy Education Review, 2 (2). pp. 20-45.

- Fitzgerald, M.T., Hollow, R., Rebull, L.M., Danaia, L., McKinnon, D.H., (2014), "A review of high school level astronomy student research projects over the last two decades", Publications of the Astronomical Society of Australia, 31, e037

- Gomez, E.L. \& Fitzgerald, M.T., (2017), "Robotic telescopes in education", Astronomical Review, 2017, 1

\section{Technical Articles about Robotic} Telescopes and Networks in general (26 articles)

- Baruch, John, E. F. (1992) Robots in Astronomy. Vistas in Astronomy, 35. pp. 399-438.

- Castro-Tirado, A. J. (2010) Robotic Autonomous Observatories: A 
Historical Perspective. Advances in Astronomy, 2010. pp. 1-8.

- Genet, Russell M. (2002) Automatic Photometric Telescopes: Past and Future. In: The Society for Astronomical Sciences 21st Annual Symposium on Telescope Science, held May 22-23, 2002, at Big Bear, CA. Society for Astronomical Sciences, pp. 61-66.

- Querci, F. R. and Querci, M. (2000) Robotic telescopes and networks: New tools for education and science.

Astrophysics and Space Science, 273 (1). pp. 257-272

\section{Relevant Articles about Research and} Evaluation Methodology (4 articles)

- Buxner, Sanlyn R. and Wenger, M. C. and Dokter, E. F. C. (2011) Exploring Assessment Tools for Research and Evaluation in Astronomy Education and Outreach. In: ASP Conference Series: Earth and Space Science: Making Connections in Education and Public Outreach, 31 July - 4 August 2010, University of Colorado, Boulder, Colorado.

- Brogt, E. and Dokter, E. and Antonellis, J. and Buxner, S. (2007) Regulations and Ethical Considerations for Astronomy Education Research II: Resources and Worked Examples. Astronomy Education Review, 6 (2). pp. 99-110.

\section{Broad Project Outline and Description papers (82 articles)}

- Coward, D. M. and Heary, A. and Venville, G. and Todd, M. and Laas-Bourez, M. and Zadnick, M. and Klotz, A. and Boër, M. and Longnecker, N. (2011) The Zadko telescope: A resource for science education enrichment. Advances in Space Research, 47. pp. 1922-1930.

- Gould, Alan (2010) Transits, Binaries, and Hands-On Universe. In: ASP Conference Series: Science Education and Outreach: Forging a Path to the Future, 12-16 September 2009, Millbrae, California, USA.

- Kadooka, M. A. and Meech, K. J. and Bedient, J. (2002) TOPS Telescope Projects on Variable Stars and Other Objects. Journal of the American Association of Variable Star Observers (JAAVSO), 31 (1). pp. 39-47.

- Pennypacker, C. and Barclay, T. (2003) Building a Global Education and Science System Based on Modest-Aperture Telescopes: The Hands-On Universe System. In: The Future of Small Telescopes in the New Millennium. Astrophysics and Space Science Library, 2 . Springer (Kluwer Academic Publishers), Netherlands, pp. 121-126.

- Rebull, Luisa M. and Gorjian, V. and Squires, G. (2012) Authentic Astronomy Research Experiences for Teachers: The NASA/IPAC Teacher Archive Research Program (NITARP). In: Connecting People to Science. ASP Conference Series.

\section{Papers about instrumentation and software (17 articles)}

- Brown, T.M. and Baliber, N. and Bianco, F.B. et al. (2013) Las Cumbres Observatory Global Telescope Network. Publications of the Astronomical Society of the Pacific, 125. pp. 1031-1055.

- Collins, K.A., Kielkopf, J.F., Stassun, K.G., Hessman, F.V., (2017), "AstroImageJ: Image Processing and Photometric Extraction for 
Ultra-Precise Astronomical Light Curves", The Astronomical Journal, 153 (77). pp. 1-13.

- Laher, Russ R. and Gorjian, Varoujan and Rebull, Luisa M. and Masci, Frank J. and Fowler, John W. and Helou, George and Kulkarni, Shrinivas R. and Law, Nicholas M. (2012) Aperture Photometry Tool. Publications of the Astronomical Society of the Pacific, 124. pp. 737-763.

- Luckas, Paul (2013) The design, construction and use of an Internet accessible, robotic optical telescope initiative for student research projects. Masters thesis, University of Western Australia.

\section{Project papers with some basic student data ( 25 articles)}

- Dussault, M. and Reinfeld, E. and Sienkiewizcz, F. and Deutsch, F. and Fruchtman, S. and Gould, R. and Smith, D. A. and Eisenhammer, B. (2010) Observing With NASA: Using Online Telescopes to Expand the Pipeline of Astronomy Learners. In: ASP Conference Series: Science Education and Outreach: Forging a Path to the Future, 12-16 September 2009, Millbrae, California, USA.

- Rosen, R. and Heatherly, S. and McLaughlin, M. A. (2010) The Pulsar Search Collaboratory. Astronomy Education Review, 9 (1). pp. 1-13.

- Hollow, R. and Hobbs, G and Champion, D and Amy, S. and Khoo, J and Chapman, $\mathrm{J}$ and Mulcahy, $\mathrm{M}$ and Alem, L. and Krumm-Heller, A. and McKinnon, David H. and Danaia, Lena J. and Jenet, F and Carr, M (2008) PULSE@ Parkes: Pulsar Observing for High School Students. In: ASP Conference Series: Preparing for the
2009 International Year of Astronomy: A Hands-On Symposium, 1-5 June, 2008, St. Louis, Missouri, USA.

\section{Deeper education research with RTSRE projects (32 articles)}

- Etkina, E., Matilsky, T., Lawrence, M., (2003), "Pushing to the Edge: Rutgers Astrophysics Institute Motivates Talented High School Students", Journal of Research in Science Teaching, 40 (10). pp. 958-985.

- Richwine, P.L., (2007), "The Impact of Authentic Science Inquiry Experiences Studying Variable Stars on High School Students' Knowledge and Attitude about Science and Astronomy and Beliefs Regarding the Nature of Science", Doctoral thesis, University of Arizona.

- Wilson, J.W., (2003), "Science Teachers Learning About the Nature Of Science and Scientific Inquiry by Doing Astronomical Research: The Binary Star Project", Doctoral thesis, Georgia State University, College of Education.

\section{Theoretical or Position Papers (49 articles)}

- Slater, T. F. and Burrows, A. C. and French, D. A. and Sanchez, R. A. and Tatge, C. B. (2014) A Proposed Astronomy Learning Progression For Remote Telescope Observation. Journal of College Teaching \& Learning, 11 (4). pp. 197-206.

- Percy, J.R., (2008), “Learning Astronomy by Doing Astronomy", In: Innovation in Astronomy Education. Cambridge University Press, pp. 13-22.

- Hollow, Robert (2000) The Student as Scientist: Secondary Student Research Projects in Astronomy. Publications of 


\section{A Brief Overview of Robotic Telescopes, Student Research and Education Research in the \\ English Literature - 7}

the Astronomical Society of Australia, 17 (2). pp. 162-167.

The broad distribution of these articles is shown in Table 1.

Table 1. Distribution of major types of publications in the RTSRE literature

\begin{tabular}{|c|c|}
\hline & \# of Articles \\
\hline Broad Reviews & 10 \\
\hline Technical articles & 26 \\
\hline Research/Evaluation Methods & 4 \\
\hline Broad project outlines/Descriptions & 82 \\
\hline Instrumentation and Software & 17 \\
\hline Project papers with some data & 25 \\
\hline Full Education Research & 32 \\
\hline Theoretical/Position Papers & 49 \\
\hline
\end{tabular}

There are no known external guidelines as to what the distribution of 'types' of publication should be, so much of this discussion is speculative. It can be seen that by far and away the most common type of article is a broad project outline or description following by theoretical papers and statements of position, the commonality here being these papers do not need significant data to generate. The least common are papers on Research and/or Evaluation methods. The articles classified as "Full Education Research" also largely contain most of the 22 theses in this study; hence the number of non-thesis publications of this type is also quite low. The commonalities here between these two types being that these papers are either about data or require significant data for publication. Hence, despite the lack of external guidelines as to what to expect, it seems reasonable to state from this that publications relying heavily on robust data are currently lacking in this literature.

\section{The Major Locations of Publication}

The 245 articles identified in this study were published in 61 different places including books, journals, theses and conference proceedings. If this were evenly spread across the field, that would equate to roughly 4 articles published per location.
This isn't the case. Only in 12 locations did the RTSRE field publish more than 5 times, usually many more, with the rest ( 6 book chapters, 24 conference proceedings, 40 Journal Articles), distributed in an uncollected manner across 49 different locations. The choice of 5 articles as an indicator for a 'major location' was a combination of this being above the average number of articles per location and being the bare minimum number of articles where simple statistics (albeit small in some cases) become meaningful. The major publication locations are outlined in Table 2 . It can be seen that the ASP Conference Series holds the most articles in the field. 60 different articles or about a quarter of the entire literature. This depends on your perspective however as these 60 articles were distributed across 15 separate conference proceedings booklets. There are only four of these conference proceedings volumes which had more than five articles:

Vol. 89 (10 articles): Astronomy Education: Current Developments, Future Coordination (1996)

Vol. 220 (7 articles): Amateur-Professional Partnerships in Astronomy (2000)

Vol. 431 (6 articles): Science Education and Outreach: Forging a path to the future (2010) Vol. 443 (6 articles): Earth and Space Science: Making Connections in Education and Public Outreach. (2011)

The AIP Conference Series collects articles from a multitude of physics conferences around the world. There isn't any relationship between the articles, there just has been a lot of relevant conferences attached to the AIP. The "American Journal of Physics," despite its name, is not really a physics research journal but rather a physics teacher association professional journal. There are a number of interesting articles there but they do tend to be focused on descriptions of lab or practical activities rather than academic articles although there is a small Physics Education Research section in this journal.

The book, "Astronomical Education with the Internet" (Okyudo et al., 1998), contains 17 


\section{A Brief Overview of Robotic Telescopes, Student Research and Education Research in the English Literature - 8}

Table 2. Locations where more than 5 articles were published. Frequency refers to the number of articles, while Percent refers to the percentage of the entire literature this represents.

\begin{tabular}{|c|c|c|}
\hline & Frequency & Percent \\
\hline Other & 70 & 28.6 \\
\hline ASP Conference Series & 60 & 24.5 \\
\hline Thesis & 22 & 9 \\
\hline Astronomical Education with the Internet & 17 & 6.9 \\
\hline The Future of Small Telescopes in the New Millennium & 15 & 6.1 \\
\hline jAAVSO & 12 & 4.9 \\
\hline Publications of the Astronomical Society of Australia & 11 & 4.5 \\
\hline AIP Conference Proceedings & 7 & 2.9 \\
\hline American Journal of Physics & 7 & 2.9 \\
\hline Astronomy Education Review & 7 & 2.9 \\
\hline Innovation in Astronomy Education & 6 & 2.4 \\
\hline Telescopes from Afar Conference & 6 & 2.4 \\
\hline Publications of the Astronomical Society of the Pacific & 5 & 2 \\
\hline
\end{tabular}

individual chapters which are included in the RTSRE literature. It arose out of a Japanese conference in June 1998, which drew a quite impressive collection of articles from a strong collection of early pioneers in the field. While there are many articles overall, not many of them are very substantial articles. "Astronomy Education Review" (Fraknoi 2014; Fraknoi and Wolff 2001) was an astronomy education journal initially run through NOAO but towards the end run by the AAS who decided to cease publication in 2013. It did form a core journal of the field during this period but also suffered from a number of issues related to impact factor, indexing and the typical academic level of the publications. As it was a core journal, many outline articles for RTSRE projects were published there during the period of its existence. "Innovation in Astronomy Education" was a 2008 book (Pasachoff et al., 2008) formed from the proceedings of the 2006 Special Session of the International Astronomical Union General Assembly in Prague.

"jAAVSO" is the journal of the American Association of Variable Star Observers. It publishes articles related to variable star astronomy including articles on education research and historical articles.. The "Publications of the Astronomical Society of Australia" is what it says, it is a mainline professional academic astronomy journal with the marvelous twist that it is open to accepting astronomy education articles (as well as other more leftfield topics) which makes it one of the few mainstream astronomy research journals in this list. The "Publications of the Astronomical Society of the Pacific" is the other mainstream astronomy research journal which made its way into the list primarily because of important instrumental papers for Robotic Telescopes rather than due to Student Research or Education concerns.

"Telescopes from Afar", a conference in Hawai'i in 2011 on remotely operated, automated and robotic ground based telescopes, also has a number of relevant articles. The book "The Future of Small Telescopes in the New Millennium" (Oswalt, T. (Ed), 2003) is an impressive three-volume book set of hundreds of articles surrounding small telescope issues. The articles from the set are all written by key players in the field on interesting topics. The last category "Thesis" is typically a Masters or $\mathrm{PhD}$-level thesis on RTSRE topics. It is usually here that you can find questions being explored to great depth, even more so than a journal article. While this location of thesis publication is not a traditional book, journal or proceedings with a single ISBN or ISSN, it is important to illustrate 


\section{A Brief Overview of Robotic Telescopes, Student Research and Education Research in the \\ English Literature - 9}

what fraction of the research in this field is published predominantly in thesis form at various institutions. Most of the theses were published in the USA (16) with West Virginia University and the University of Arizona both publishing three theses on RTSRE topics with the others published in Australia (5) and the United Kingdom (1).

\section{Impact Analysis of Publications}

Currently the impact of a research article, a journal or an actual researcher is usually quantified into some type of metric usually involving the number of papers and the number of citations to individual papers. One of the most popular journal-focussed measures of quality is the journal 'impact factor' which is the number of citations in the previous two years to all articles divided by the number of articles published. So, very roughly, how many citations per year per article. We will use a similar metric to measure impact as outlined below. We have not considering the journal 'impact factors' provided by Scopus or ISI as these are representative of works within the journals which are largely not RTSRE-related articles.

The current most commonly used index in the current era for an individual researcher is the "h-index" (Hirsch, 2005) This is a metric that measures a researcher's impact by what is the nth paper of a list sorted (large to small) by the author's citations that has n number of citations. This is the metric most used to estimate an individual academic's research output by publications and citations alone but that is likely to change soon as indices become more sophisticated.

Citations can be perceived as a pawn in a numbers game to forward one's academic career by displaying a high number of citations (or a h-index resulting from the citations) on a $\mathrm{CV}$ in order to get the next grant or position. This is more true than it should be. Once citations became a measure of research impact, by Goodhart's Law - "When a measure becomes a target, it ceases to be a good measure" - it loses its power to measure impact. In this paper, we approach citations just to see who has been citing who, why, where and for what reason rather than necessarily using "citation impact" as a competitive rating scale.

To analyse the impact of the papers in the field, the number of citations per year since publication, as measured by Google Scholar, were calculated where the maximum number of years in the denominator is 7 . The choice of 7 was a rough estimate of the reach in years of the impact of an average paper before it would typically slowly stop being cited. This maximum figure was to prevent older articles being artificially devalued in the metric. If an article older than 7 years is still collecting citations, then it should probably collect some bonus points anyway.

A hypothetical, but authentic, example of this might be that a robotic telescope project may have begun in the 1991 and published a paper about their work in 1993. This is early days for the field and perhaps they had some success that people in their era found useful (and citable). It does seem reasonable that continuing throughout the 1990s this project would be cited by similarly orientated projects as examples and comparisons of where they are heading and what has been done before. It seems unlikely, given the pace of technological progress and typical academic citation practices, that projects beginning in the 00's would tend to cite such a paper.

These 00's projects start from an entirely different level of capacity and approach. Hence, if we were not to impose a limit on the denominator (years), the important early 1990s paper would be much diminished in estimated impact (even though the nominator - citations - have stopped, the denominator - years since publication - continues to increase), even though it may be a seminal work in the field. To be entirely fair to older papers, there should be a correction factor considering that there were less people working in the field in the early 1990s than now and hence less authors to cite their work. It is not clear how this correction factor could be estimated and, hence, has not been attempted in this work.

This metric for estimated impact may seem to be a little arbitrary, simplistic and rough. We cannot do 


\section{A Brief Overview of Robotic Telescopes, Student Research and Education Research in the \\ English Literature - 10}

any better though as our literature is dispersed through, not only multiple journals and proceedings but, multiple different fields of research. Different fields of research have different publication cultures. For instance, the average number of authors on an education article is about three, whereas for astronomy, it is usually in the tens of authors. This alone, after a relatively small amount of thought, should lead us to expect that publication impact metrics between the two fields should be significantly different.

According to the Web of Science metric (Harzing and Alakangas, 2016), for professors at equivalent stages of their career, social scientists will have 30 authored papers, whereas natural scientists will have 98 (humanities have 16!). The number of citations a social scientist will have is 591 , whereas a natural scientist will have 2612 (humanities have 61 !). The h-index of a social scientist would be 9.6, whereas a natural scientist will be 25.6 (humanities have 3.5!). If different indices are examined (e.g. Scopus or Google Scholar), different values are estimated. Having said that, as can be seen in Figure 2, the median cites/year by field is fairly equivalent at roughly 0.2 citations per paper per year regardless of whether the field was Astronomy, Astronomy Education or Education.

In Figure 3, the histogram of citations per year for all RTSRE-related publications is shown. It can be seen that the mode of the distribution is quite clearly not far above zero. Most articles do not get cited. They have been written and they have never been referred to or used in further studies. More disturbingly, because Google Scholar is being used here here, which does not remove self-citations, this means that not only have other people not cited this work, but that the author of these articles never wrote another article that referred back to their own work.

In Figure 4, showing the citation rates for the major locations of publication, we can see that the academic peer reviewed journals, AER, AJP, PASA and PASP clearly have the highest citation rates. This is to be suspected as these are academic journals where there is a culture of referencing and citing and an appreciation of the published work. It is also likely to be held to be a more trusted source and there is a much higher bar to entry to being published in terms of quality but also in terms of being a substantial contribution to the field. Hence, it is likely that papers that would not be cited would probably not be accepted for publication. The pro-am peer-reviewed journal, jAAVSO, doesn't cite as highly as the other journals even though there is a significant amount of student research published in there.

In Figure 5, we show the citation rate distributions for books, conference proceedings, journals and thesis. As noted just recently, it is very clear that journals (at least in terms of citations) outperform book chapters, thesis and conference proceedings. Theses are marginally higher in median and significantly higher in range than books and proceedings, usually being a more substantial work with greater size and depth. They would be higher but journal articles are typically generated from the thesis which tends to shift the location of future citation away from the thesis towards the journal article. This leaves books and conference proceedings as the under-performers.

Books hold an interesting position in academia. They are held in high regard in education, in very high regard in the humanities, but not so highly in astronomy. It is certainly true that the book chapters examined in this study seem to be thoroughly and carefully written, of great interest, and seem worthy of much higher citation rates (compared to equivalent journal articles) than they actually have achieved. They are also, however, problematic to access. The books in this review had to be accessed by visiting physical libraries at multiple institutions, accessing some online through multiple institutional subscriptions and, in one case, tracking down an individual who still had a copy of the book. The books that are in the istardb abstract database were hard to source and there are still a few older, rarer, books still to be tracked down to be examined.

Part of the reason that, despite their high intrinsic rating by scholars, these books have such a low 


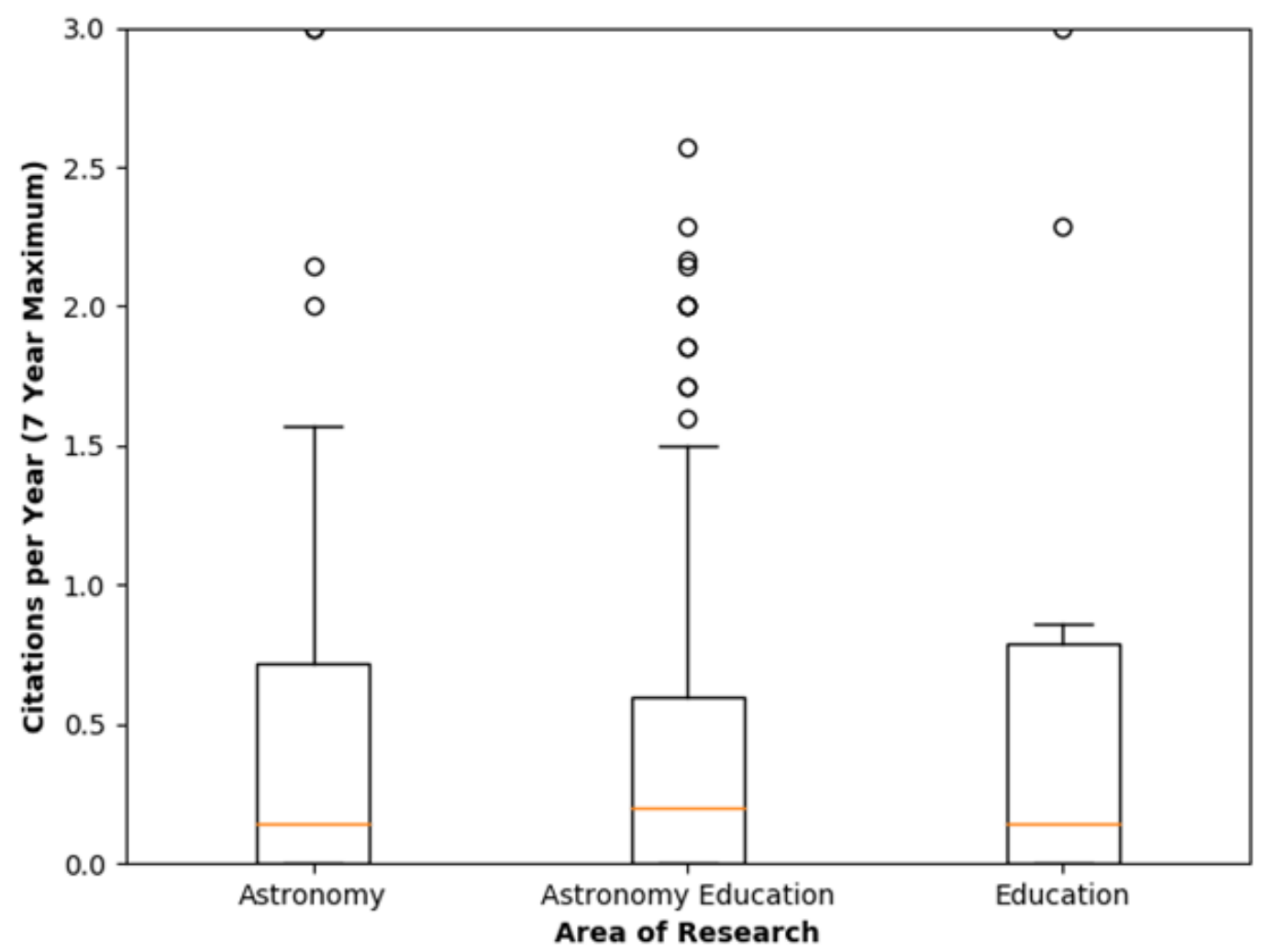

Figure 2. Box-plot representation of the distribution of citation rates for the fields of Astronomy, Astronomy Education, and Education in the RTSRE literature. (Orange line indicates median of the distribution, the box represents the lower to upper quartile values of the data, the whiskers extend to the last data point beyond $1.5 *$ the Interquartile Range, circles represent outliers beyond this range)

citation rate is potentially due to this lack of accessibility. It could be said that most education journals also require institutional subscriptions (while astronomy journals usually are Open Access after two years or authors provide their preprints on arxiv) and are somewhat inaccessible. However, RTSRE publications (thus far) are rarely in the mainstream science education journals anyway, although this may change over time as the field becomes methodologically more robust and diverse. The accessibility of these journals is offset by the fact that most institutions, nearly by default, seem to have access to the major education journals of interest.

Conference Proceedings, on the other hand, do not suffer such a lack of accessibility. Most

proceedings are available freely online and are indexed well (at least in Google Scholar). However, their citation rates are equally as low as books (albeit with a few outliers). It should also be noted that the Astronomical Society of the Pacific Conference Series, making up a $24.5 \%$ of the field's publications, has $5.7 \%$ of the citations with a median citation rate of 0 cites per year per paper.

\section{Citation Rates Related to Article Contents}

This is another area where there is some amount of bias and a fuzzy measurement. All of the articles have been read by the first author and, acknowledging a single-rater bias inherent in this approach, the articles were rated on two different metrics according to the depth of data used in the paper and the depth of discussion about issues in the paper.

In Figure 6, the citation rate as a function of the amount of data in the paper is presented. Broadly speaking, this figure indicates that the more data in 


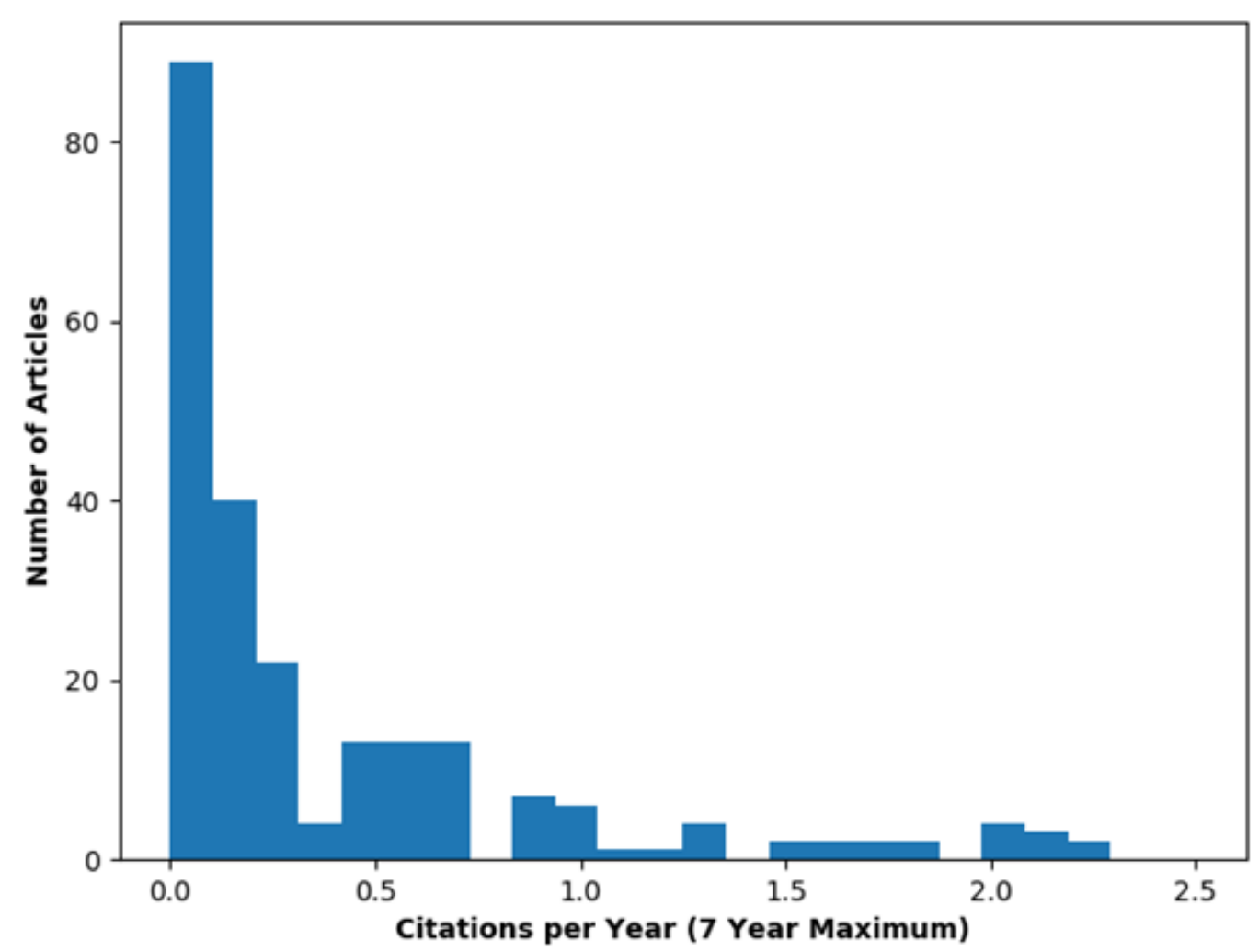

Figure 3. Histogram of the citation rates of articles in the RTSRE field.

the paper and the more robust the data, the more useful others will find the paper and the higher the impact of the paper. There are outliers for "This is what we will do" and "This is what we do" papers as sometimes people actually do something more significant and this, while lacking in data, becomes a seminal paper for the project. The graph provides clear advice: "Aim to incorporate data from as robust a methodology into your paper where possible".

Figure 7, which presents the citation rate of papers as a function of their analysis and discussion of the data, also presents something fairly straightforward. Simply describing what happens, or what will happen, does not attract many citations. What other researchers and practitioners are likely going to be looking for is advice for their projects (what worked/didn't work) and especially the reasons "why" things work and do not work so that they can adapt this to their own context. That things happen or do not happen just by themselves is not of great interest (as measured by citations). The advice here is simple: "Try to analyse and discuss your data or project in terms of why things work and do not work with reference to some type of underlying theory".

\section{Where to Publish?}

Conference Proceedings are definitely worth the trouble. It is a place where an enduring record of your contribution to the conference can be set in digital stone. It should form an intrinsic part of every conference so that the professional and personal connections and inspiration generation hopefully continue on into the future as well as a written record of your important contribution to the field, that is connected and embedded into the larger academic, professional and practitioner literature. It should be that conference proceedings contain unique information (as actually required ethically!) that is useful (and therefore citable) in the future by yourselves and others. While considered lower in impact value than journal articles, they are also open to broader, more speculative, "work in progress" articles. 


\section{A Brief Overview of Robotic Telescopes, Student Research and Education Research in the English Literature - 13}

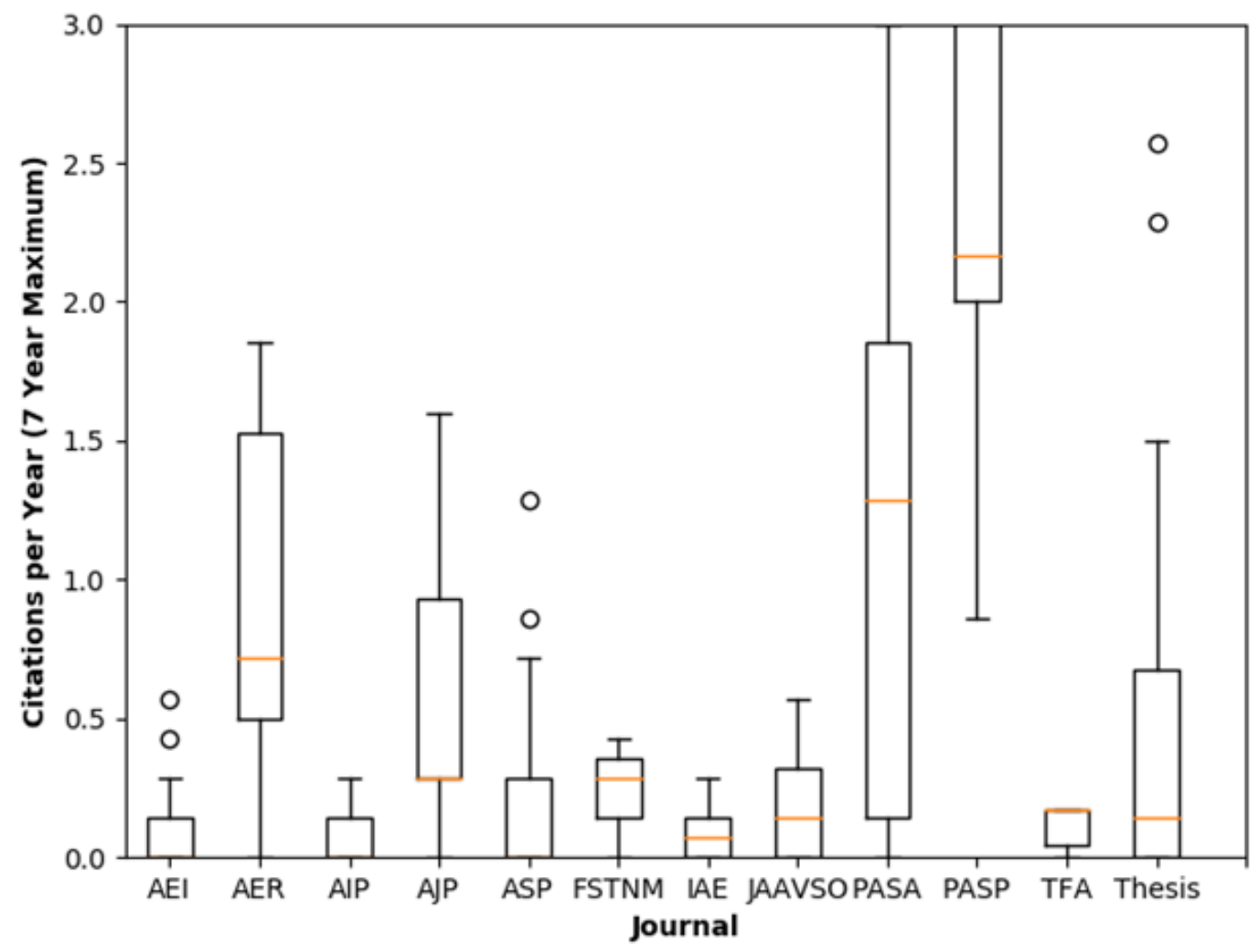

Figure 4. Box plot representation of the distribution of citation rates for major locations of publication in the RTSRE Field. (AEI: Astronomy Education with the Internet, AER: Astronomy Education Review, AIP: American Institute of Physics Conference Series, AJP: American Journal of Physics, ASP: Astronomical Society of the Pacfic, FSTNM: The Future of Small Telescopes in the New Millenium, IAE: Innovation in Astronomy Education, jAAVSO: Journal of the American Association of Variable Star Observers, PASA: Publications of the Astronomical Society of Australia, PASP: Publications of the Astronomical Society of the Pacific, TFA: Telescopes from Afar Conference Proceedings, Thesis: PhD or Masters Thesis publication). (Boxplot ranges defined as described in Figure 2. Those that appear to not have orange lines (AEI, AIP and ASP) have median citation rates of 0.0)

Unfortunately, if we are to take the citations seriously and also consider the accessibility issue, books do not tend to be a good way to distribute information far and wide. It is also recommended that you think about the accessibility of any *journal* you might publish in. Keep in mind that most people in the world are outside of the USA, UK, Europe or Australia and have dramatically lower incomes from which to pay for subscriptions. Accessibility is also a consideration for Masters or $\mathrm{PhD}$ Thesis. While these studies are deep and represent a person's intense focus for multiple years, they do tend to end up in institutional repositories from which it may be hard to retrieve the thesis itself. There are, of course, many other considerations to think about as to whether to embark on a thesis!

Academic journals are obviously the highest impact places to publish and this is where your highest quality, most rigorous, most well-thought-out studies should be submitted. But for an RTSRE-type article, Where should this be? The most obvious location, before its final issue in 2013, would have been Astronomy Education Review, although it did draw criticism due to the relatively low quality of articles and its low impact factor in general... at least it was an obvious location that everyone in the community read. The American Journal of Physics, whilst containing a Physics Education Research section, isn't purely 


\section{A Brief Overview of Robotic Telescopes, Student Research and Education Research in the English Literature - 14}

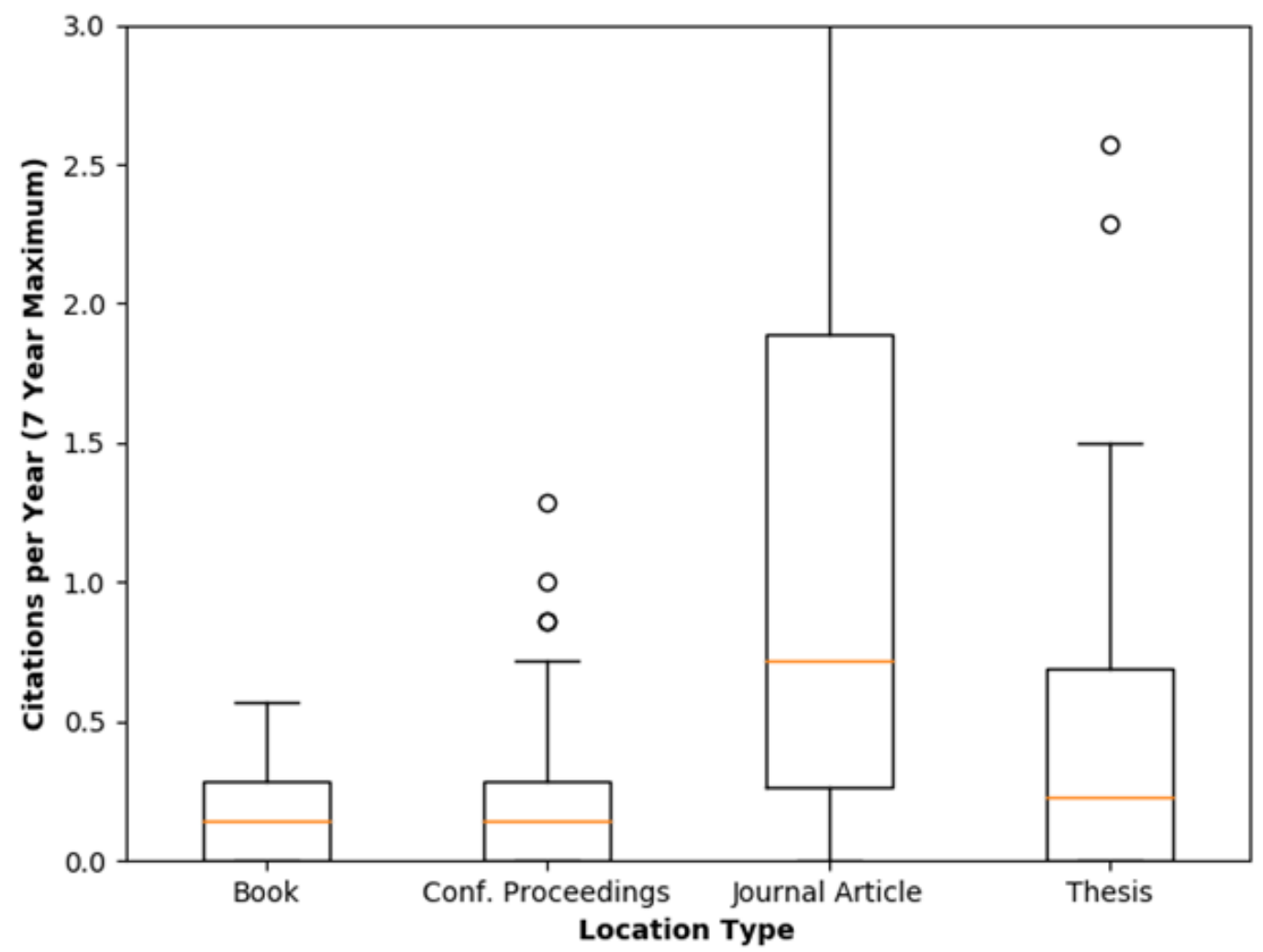

Figure 5. Box plot representation of the distribution of citation performance by publication type. (Boxplot ranges defined as described in Figure 2)

aimed at being a scholarly academic journal where citations are a core, but rather as a place to publish articles that are of interest to a "diverse audience of physics students, educators and researchers".

Despite this, the journal has had a median citation rate that has only been bettered by Astronomy Education Review, Publications of the Astronomy Society of Australia and Publications of the Astronomy Society of the Pacific and may be worth examination as a potential place for publication.

There are a few journals emerging that may be good "Astronomy Education" journals to submit to. The most obvious is the "Journal of Astronomy and Earth Sciences Education" or JAESE (Slater, 2014), a relatively new journal (the first issue was in 2014) The Physical Review Physics Education Research journal at publication of this article is going through the process of an Astronomy Education Research special issue and welcomes astronomy education research articles at any time. Another "Astronomy Education" option is the
"Latin-American Journal of Astronomy Education" (RELEA) (Bretones et al., 2016). It may be noted that none of these three journals rose above the 5 article threshold to be on the major RTSRE publication locations list, but they may in future.

The three astronomy journals that rose above the 5 article threshold were jAAVSO, Publications of the Astronomical Society of Australia (PASA) and the Publications of the Astronomical Society of the Pacific (PASP). Each of them are certainly worthy journals in their own right. PASA is the most unique in that it is an astronomical journal that is in the top quartile of impact factors for astronomy journals that also accepts astronomy education articles. PASP rose into the major journal list primarily because it had some highly cited, but more technical, articles (such as the Las Cumbres Observatory paper of Brown et al. 2013 and the Aperture Photometry Tool paper of Laher et al. 2012). The jAAVSO is a journal for pro-am variable star collaboration papers, education 


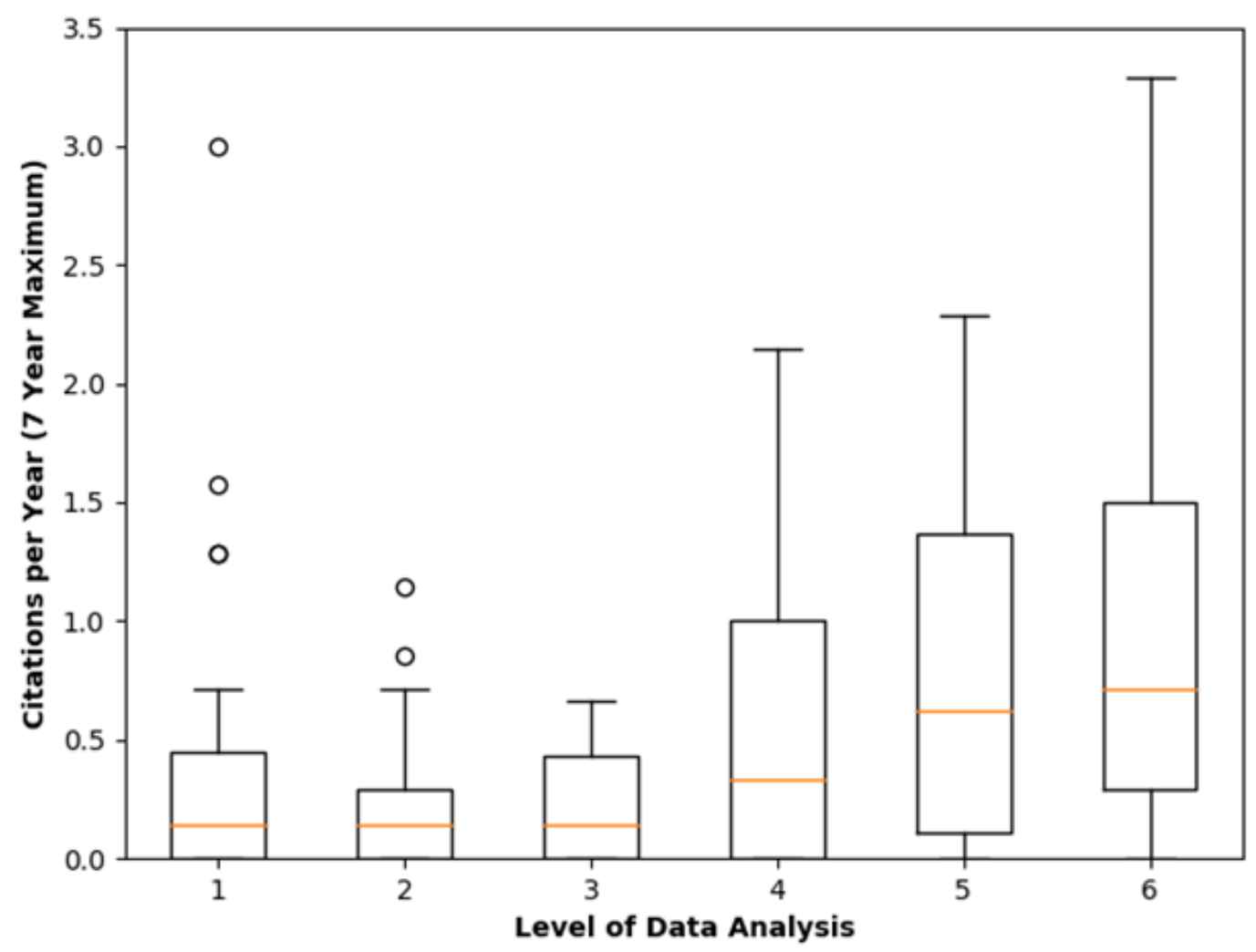

Figure 6. Box plot representation of the distribution of citation rates by level of data analysis used in the paper. (1) "This is what we will do", (2) "This is what we do", (3) "This is what we do with some demographical/context data", (4) "This is what we do with anecdotes and shallowly analysed results", (5) "This is what we do with some imperfect but usable data", (6) "This is what we do with robust data methodology". (Boxplot ranges defined as described in Figure 2)

research and historical papers and highly recommended if your project is along these lines.

It is a very good idea to aim for Open Access for our articles, especially in an emerging field. It is a coincidence, but a nice coincidence, that the journals that emerged as strong in the field as well as the journals that have potential in the field are Open Access at some point. PASA, PASP and jAAVSO are Open Access after two years. JAESE, PRPER and RELEA are open access from the start. This is important. A substantial fraction of RTSRE field members are not linked to institutions and cannot benefit from institutional journal subscriptions. It is also true that outside the USA/Europe world institutions from those areas (especially China, S. Korea, India and the other South to South-East Asian countries) likely cannot afford journal subscriptions, but it is very likely that Asia will be the major growth area in the field over the next 5-10 years.

\section{Closing Comments}

Nobody can expect what will come from publication. You might spend many long late nights writing an article that nobody will ever read and it may evaporate into academic air (no comment). Other articles, such as the 2014 review of student research projects (Fitzgerald et al., 2014), may start off a chaotic butterfly effect chain of events where you blink and all of a sudden you've helped hold a really successful fun conference (RTSRE).

What is decidedly true, is that if you never publish, then nobody will ever know what you did. Humans, being the fickle creatures they are, in the absence of evidence will likely assume you did nothing. Therefore, record what you did via publication. In recording what you did, acknowledge other 


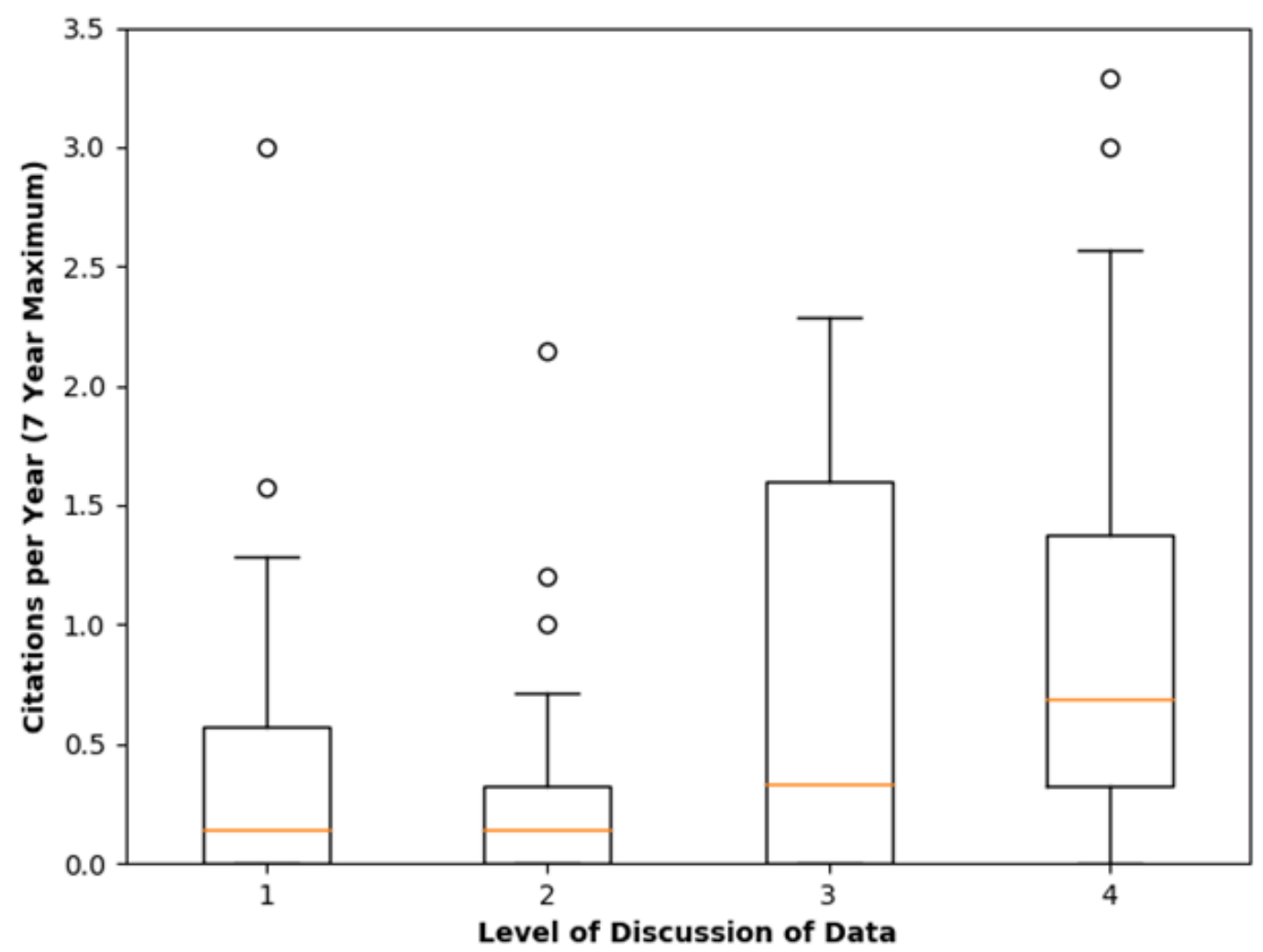

Figure 7. Box plot representation of the distribution of citation rates in terms of level of discussion and analysis of data. (1) "This is what will happen", (2) "This is what happened", (3) "This is what worked/didn't work”, (4) "This is what worked/didn't work and why”. (Boxplot ranges defined as described in Figure 2)

peoples' work through citing their publications. Sometimes that other person is actually yourself from the past. While you may have developed as a human being, that previous version of yourself deserves credit too.

When writing your article, think about how you can add value to your paper for the readers. Initially, by being as bluntly honest as you can. Say where you have failed and why so that you may help steer others away from the wrong path. Explore in detail the problems you have encountered along the way and what solutions you might be trying and how well they worked. Try, as far ahead of time as possible, to rigorously test out your endeavors using a robust methodology and good data, especially as this field seems to be currently lacking such studies. Once you have published, try to promote your research to the field as much as possible so that conversations can begin and become enriched by your contributions to the field.

\section{References}

Asbell-Clarke, J., Pack, H., Pennypacker, C., and Toler, D. (1996). Hands-on universe: bringing astronomical explorations to the classroom. In Astronomy Education: Current Developments, Future Coordination, volume 89, page 158.

Bailey, J. M. (2011). Astronomy education research: Developmental history of the field and summary of the literature. Commissioned paper for the National Research Council Board on Science Education's Committee on the Status, Contributions, and Future Directions of Discipline Based Education Research.

Bailey, J. M. and Lombardi, D. (2015). Blazing the trail for astronomy education research. Journal of Astronomy and Earth Sciences Education, 2(2):77. 


\section{A Brief Overview of Robotic Telescopes, Student Research and Education Research in the English Literature - 17}

Bailey, J. M. and Slater, T. F. (2003). A review of astronomy education research. Astronomy Education Review, 2(2).

Baruch, J. E. (1992). Robots in astronomy. Vistas in Astronomy, 35:399-438.

Bretones, P. S., Jafelice, L. C., and Horvath, J. E. (2016). Ten years of Latin-American journal of astronomy education RELEA: achievements and challenges for international astronomy education development. Journal of Astronomy and Earth Sciences Education, 3(2):110.

Brogt, E., Dokter, E., Antonellis, J., and Buxner, S. (2008). Regulations and Ethical Considerations for Astronomy Education Research II: Resources and Worked Examples. Astronomy Education Review, 6(2):99-110.

Brown, T., Baliber, N., Bianco, F., Bowman, M., Burleson, B., Conway, P., Crellin, M., Depagne, É., De Vera, J., Dilday, B., et al. (2013). Las Cumbres Observatory global telescope network. Publications of the Astronomical Society of the Pacific, 125(931):1031.

Buxner, S., Wenger, M., and Dokter, E. (2011). Exploring Assessment Tools for Research and Evaluation in Astronomy Education and Outreach. In Earth and Space Science: Making Connections in Education and Public Outreach, volume 443, page 125 .

Castro-Tirado, A. J. (2010). Robotic autonomous observatories: a historical perspective. Advances in Astronomy, 570489.

Clark, G. (1998). Telescopes in education (TIE): Bringing live astronomy to the classroom utilizing remotely controlled telescopes. Astronomical Education with the Internet, pages 37-44.

Collins, K. A., Kielkopf, J. F., Stassun, K. G., and Hessman, F. V. (2017). AstroImageJ: image processing and photometric extraction for ultraprecise astronomical light curves. The Astronomical Journal, 153(2):77.
Coward, D., Heary, A., Venville, G., Todd, M., LaasBourez, M., Zadnik, M., Klotz, A., Boër, M., and Longnecker, N. (2011). The Zadko telescope: A resource for science education enrichment. Advances in Space Research, 47(11):1922-1930.

Danaia, L., McKinnon, D., Parker, Q., Fitzgerald, M., and Stenning, P. (2012). Space to grow: LCOGT.net and improving science engagement in schools. Astronomy Education Review, 11(1).

Dussault, M., Reinfeld, E., Sienkiewizcz, F., Deutsch, F., Fruchtman, S., Gould, R., Smith, D., and Eisenhammer, B. (2010). Observing With NASA: Using Online Telescopes to Expand the Pipeline of Astronomy Learners. In Science Education and Outreach: Forging a Path to the Future, volume 431, page 351.

Etkina, E., Matilsky, T., and Lawrence, M. (2003). Pushing to the edge: Rutgers astrophysics institute motivates talented high school students. Journal of Research in Science Teaching, 40(10):958985.

Fitzgerald, M. T., Hollow, R., Rebull, L. M., Danaia, L., and McKinnon, D. H. (2014). A review of high school level astronomy student research projects over the last two decades. Publications of the Astronomical Society of Australia, 31.

Fraknoi, A. (2014). A brief history of publishing papers on astronomy education research. Journal of Astronomy and Earth Sciences Education, 1(1):37.

Fraknoi, A. and Wolff, S. (2001). Welcome to Astronomy Education Review. Astronomy Education Review, 1(1).

Genet, R. M. (2002). Automatic Photometric Telescopes: Past and Future. In Society for Astronomical Sciences Annual Symposium, volume 21, page 61 .

Genet, R. M. (2011). History of Robotic and Remotely Operated Telescopes: The Fairborn Observatory 1979-1989. In Conference Proceedings from Telescopes from Afar. 


\section{A Brief Overview of Robotic Telescopes, Student Research and Education Research in the \\ English Literature - 18}

Gomez, E. L. and Fitzgerald, M. T. (2017). Robotic telescopes in education. Astronomical Review, 13(1):28-68.

Gould, A. (2010). Transits, binaries, and handson universe. In Science Education and Outreach: Forging a Path to the Future, volume 431, page 61 .

Harzing, A.-W. and Alakangas, S. (2016). Google Scholar, Scopus and the Web of Science: a longitudinal and cross-disciplinary comparison. Scientometrics, 106(2):787-804.

Hirsch, J. E. (2005). An index to quantify an individual's scientific research output. Proceedings of the National academy of Sciences of the United States of America, 102(46):16569.

Hollow, R., Hobbs, G., Champion, D., Amy, S., Khoo, J., Chapman, J., Mulcahy, M., Alem, L., Krumm-Heller, A., McKinnon, D., et al. (2008). PULSE@ Parkes: Pulsar Observing for High School Students. In Preparing for the 2009 International Year of Astronomy: A Hands-On Symposium, volume 400, page 190.

Kadooka, M. A., Meech, K. J., and Bedient, J. (2002). TOPS telescope projects on variable stars and other objects. Journal of the American Association of Variable Star Observers (JAAVSO), 31:39-47.

Laher, R. R., Gorjian, V., Rebull, L. M., Masci, F. J., Fowler, J. W., Helou, G., Kulkarni, S. R., and Law, N. M. (2012). Aperture photometry tool. Publications of the Astronomical Society of the Pacific, 124(917):737.

Lelliott, A. and Rollnick, M. (2010). Big ideas: A review of astronomy education research 19742008. International Journal of Science Education, 32(13):1771-1799.

Lubin, P. and van der Veen, J. (1992). The remote access astronomy project: an example of a university/high school cooperative effort. Education and Computing, 8(1-2):79-82.
Luckas, P. (2013). The Design, Construction and Use of an Internet Accessible, Robotic Optical Telescope Initiative for Student Research Projects. Master's thesis, University of Western Australia.

Okyudo, M., Ebisuzaki, T., and Nakayama, M. (1998). Astronomical Education with the Internet. In Astronomical Education with the Internet, volume 27.

Oswalt, T. (Ed) (2003). The Future of Small Telescopes In The New Millennium. 189.

Pasachoff, J. M., Ros, R. M., and Pasachoff, N. E. (2008). Innovation in astronomy education. Cambridge University Press.

Pennypacker, C. and Barclay, T. (2003). Building a Global Education and Science System Basec on Modest-Aperture Telescopes: The Hands-On Universe System. In The Future of Small Telescopes in the New Millennium, pages 421-426. Springer.

Percy, J. R. (2008). Learning astronomy by doing astronomy. Innovation in Astronomy Education, 2:13-22.

Querci, F. R. and Querci, M. (2000). Robotic telescopes and networks: New tools for education and science. Astrophysics and Space Science, 273(1-4):257-272.

Rebull, L., Gorjian, V., Squires, G., and Team, N. (2012). Authentic Astronomy Research Experiences for Teachers: The NASA/IPAC Teacher Archive Research Program (NITARP). In Connecting People to Science: A National Conference on Science Education and Public Outreach, volume 457, page 329.

Rosen, R., Heatherly, S., McLaughlin, M., Lynch, R., Kondratiev, V., Boyles, J., Wilson, M., Lorimer, D., and Ransom, S. (2010). The Pulsar Search Collaboratory. Astronomy Education Review, 9(1). 
Sadler, P. M., Gould, R. R., Leiker, P. S., Antonucci, P. R., Kimberk, R., Deutsch, F. S., Hoffman, B., Dussault, M., Contos, A., Brecher, K., et al. (2001). MicroObservatory net: a network of automated remote telescopes dedicated to educational use. Journal of Science Education and Technology, 10(1):39-55.

Slater, S. J., Tatge, C. B., Bretones, P. S., Slater, T. F., Schleigh, S. P., McKinnon, D., and Heyer, I. (2016). iSTAR First light: Characterizing astronomy education research dissertations in the iSTAR database. Journal of Astronomy and Earth Sciences Education, 3(2):125.

Slater, T. F. (2014). Editor's Note: JAESE's Initial Aim, Scope And Business Model. Journal of Astronomy and Earth Sciences Education, 1(1).

Slater, T. F., Burrows, A. C., French, D. A., Sanchez, R. A., and Tatge, C. B. (2014). A proposed astronomy learning progression for remote telescope observation. Journal of College Teaching \& Learning (Online), 11(4):197. 